\title{
Upregulation of SOX4 antagonizes cellular senescence in esophageal squamous cell carcinoma
}

\author{
RONGFEI HAN ${ }^{1,2^{*}}$, SHIYING HUANG ${ }^{1 *}$, YONGHUA BAO ${ }^{2}$, XIN LIU $^{3}$, XIAOYU PENG $^{4}$, ZHIGUO CHEN $^{5}$, \\ QIAN WANG $^{2}$, JIAQI WANG ${ }^{5}$, QIUPING ZHANG ${ }^{6}$, TIANFU WANG ${ }^{1}$, DUO ZHENG $^{1}$ and WANCAI YANG ${ }^{5}$ \\ ${ }^{1}$ Department of Basic Medicine, School of Medicine, Shenzhen University, Shenzhen, Guangdong 518060; \\ ${ }^{2}$ Department of Immunology, School of Basic Medicine, Xinxiang Medical University, Xinxiang, \\ Henan 453003; ${ }^{3}$ Department of Surgery, Guangde People's Hospital, Xuancheng, Anhui 242200; \\ ${ }^{4}$ Department of Cardiology, The First Affiliated Hospital of Shanxi Medical University, Taiyuan, Shanxi 030001; \\ ${ }^{5}$ Department of Pathology, School of Basic Medicine, Xinxiang Medical University, Xinxiang, Henan 453003; \\ ${ }^{6}$ Department of Immunology, School of Basic Medicine, Wuhan University, Wuhan, Hubei 430071, P.R. China
}

Received June 21, 2015; Accepted June 2, 2016

DOI: $10.3892 / 01.2016 .4799$

\begin{abstract}
Senescence, a terminal cell proliferation arrest that is caused by a variety of cellular stresses such as telomere erosion, DNA damage and oncogenic signaling, is classically considered a tumor defense barrier. However, the mechanism by which cancer cells overcome senescence is undetermined. In this study, the gene expression array data of esophageal squamous cell carcinoma (ESCC) was compared with paired normal tissues and showed that a cohort of genes, including proteinases, chemokines and inflammation factors, are upregulated in ESCC, which exhibits the senescence-associated secretory phenotype. In addition, reverse transcription-quantitative polymerase chain reaction was used to demonstrate that gender determining region Y-box 4 (SOX4) is upregulated in ESCC, and that its expression is inversely correlated with senescence markers. In addition, the knockdown of SOX4 expression by short hairpin RNA decreases ESCC cell proliferation and enhances doxorubicin-induced cell senescence. These results reveal the presence of a senescent microenvironment in ESCC, and suggest an important antisenescence role of SOX4 in ESCC progression.
\end{abstract}

Correspondence to: Dr Wancai Yang, Department of Pathology, School of Basic Medicine, Xinxiang Medical University, 601 East Jinsui Avenue, Xinxiang, Henan 453003, P.R. China

E-mail:wyang06@uic.edu

Dr Duo Zheng, Department of Basic Medicine, School of Medicine, Shenzhen University, 3688 Nanhai Avenue, Shenzhen, Guangdong 518060, P.R. China

E-mail:dzheng@szu.edu.cn

*Contributed equally

Key words: esophageal squamous cell carcinoma, senescence, gender determining region Y-box 4

\section{Introduction}

Esophageal cancer is a common malignancy and is the sixth leading cause of cancer-associated mortality worldwide (1). Esophageal cancer has a striking geographical distribution; for example, with an increased prevalence in the north and central regions of China (2). Histopathologically, the vast majority of esophageal cancer cases in China are of the esophageal squamous cell carcinoma (ESCC) type (3). Currently, there are limited clinical approaches for the early diagnosis and treatment of ESCC, resulting in a 5-year survival rate of $\sim 10 \%$ for patients (3). A better understanding of the molecular events involved in the development of ESCC may offer opportunities to identify diagnostic markers, therapeutic targets or prognostic indicators for this disease.

Cellular senescence, a physiological program of irreversible growth arrest that is triggered by multiple factors such as oncogenic stress and DNA damage, is considered to be important for the development of cancer (4). Senescent cells exhibit a characteristic increase of senescence associated- $\beta$-galactosidase (SA- $\beta$-Gal) activity together with profound alterations in protein secretion that are collectively called the senescence-associated secretory phenotype (SASP) or senescence-messaging secretome (5). Among these secreted molecules, plasminogen activator inhibitor-1 (PAI1), matrix metalloproteinases (MMPs), chemokines such as chemokine (C-X-C motif) ligand 1 (CXCL1) and interleukin (IL)8, proinflammatory cytokines, including IL1 and IL6, and other molecules are involved in insulin-like growth factor (IGF), transforming growth factor- $\beta$ (TGF- $\beta$ ), tumor necrosis factor (TNF) and interferon (IFN) signaling (5-8). Senescence is a defense against potentially dangerous mutations, locking the afflicted cells into a permanent state of arrest (9). However, the mechanism by which cancer cells escape from senescence and progress to malignancy is poorly studied.

Gender determining region Y-box 4 (SOX4) is a member of the SOX transcription factor family that is characterized by a highly conserved sequence in the high-mobility group 
DNA-binding domain (10). SOX4 has been shown to be important for numerous developmental processes, including embryonic cardiac, thymocyte and nervous system development (11). SOX4 is highly upregulated in a number of human cancers, including breast cancer, hepatocellular carcinoma, colon cancer and leukemia (12-15). The expression and activity of SOX4 are regulated by various signals, including the epidermal growth factor receptor, TGF- $\beta$ and $\mathrm{Wnt} / \beta$-catenin pathways $(10,16)$. In addition, the deregulated expression of SOX4 has been shown to induce an epithelial-to-mesenchymal transition and metastasis in cancer cells (17). The present study demonstrated that SOX4 was upregulated and mediated an antisenescence effect in ESCC, thus elucidating the important role of SOX4 in the progression of ESCC.

\section{Materials and methods}

Clinical samples and cell culture. The present study was approved by the Ethics Committee of Xinxiang Medical University, Xinxiang, China. Written consent was obtained from all participants. Human ESCC tumor and adjacent non-tumor tissues were collected from 14 ESCC patients at the Anyang Tumor Hospital (Anyang, China) and frozen in liquid nitrogen. None of the patients received pre-operative chemical or radiation therapy.

ESCC gene expression microarray data was downloaded from NCBI Gene Expression Omnibus database (accession number, GSE23400; Affymetrix Human Genome U133A Array platform) (18). The Cancer Genome Atlas (TCGA) RNA-Seq data (https://tcga-data.nci.nih.gov/tcga/) was used to investigate the gene expression profile in stomach adenocarcinoma.

The human ESCC KYSE410 and KYSE510 cell lines were cultured in RPMI-1640 (Hyclone; GE Healthcare Life Sciences, Logan, UT, USA) containing penicillin (100 U/ml), streptomycin $(100 \mathrm{mg} / \mathrm{ml}$; Beyotime Institute of Biotechnology, Haimen, China), and 10\% fetal bovine serum (Hyclone; GE Healthcare Life Sciences) at $37^{\circ} \mathrm{C}$ in a humidified incubator supplemented with $5 \% \mathrm{CO}_{2}$ in air. Doxorubicin was purchased from Meilun Pharmaceutical Co., Ltd. (Dalian, China) and dissolved in double-distilled water $(1.0 \mathrm{~g} / \mathrm{ml})$ for storage and diluted with phosphate-buffered saline (PBS) prior to use.

RNA isolation and reverse transcription-quantitative polymerase chain reaction ( $R T-q P C R)$. Total RNA was isolated with TRIzol reagent (Invitrogen; Thermo Fisher Scientific, Inc., Waltham, MA, USA) according to the manufacturer's specifications, and quantified using optical density measurements from a spectrophotometer at $260 \mathrm{~nm}$. RNA $(2 \mu \mathrm{g})$ was reverse transcribed into complementary DNA (cDNA) using Moloney Murine Leukemia Virus Reverse Transcriptase (Fermentas; Thermo Fisher Scientific, Inc.) according to the manufacturer's protocol. RT-qPCR analysis was performed using SYBR Green-based detection on an ABI Step One Plus instrument (Thermo Fisher Scientific, Inc.). Following denaturation at $95^{\circ} \mathrm{C}$ for $2 \mathrm{~min}, \mathrm{qPCR}$ was performed for 40 cycles consisting of $95^{\circ} \mathrm{C}$ for $15 \mathrm{sec}, 60^{\circ} \mathrm{C}$ for $15 \mathrm{sec}$ and $72^{\circ} \mathrm{C}$ for $30 \mathrm{sec}$. Each experiment was performed three times independently. The following primers were used for qPCR: SOX4, 5'-GACCTGCTCGACCTGAACC-3' (sense) and 5'-CCGGGC
TCGAAGTTAAAATCC-3' (antisense); and glyceraldehyde 3-phosphate dehydrogenase (GAPDH), 5'-CTGGGCTACACT GAGCACC-3' (sense) and 5'-AAGTGGTCGTTGAGGGCA ATG-3' (antisense) as the control. The relative expression of the gene of interest was normalized to GAPDH and calculated with $2^{-\Delta \Delta \mathrm{Cq}}$ method (19).

Western blotting. Cells $\left(5 \times 10^{6}\right)$ in $6-\mathrm{cm}$ dishes were washed once with cold PBS and harvested by scraping into radioimmunoprecipitation assay lysis buffer on ice. Protein concentration was determined by the Bradford assay (Beyotime Institute of Biotechnology). The extracted proteins (40 $\mu \mathrm{g} /$ lane) were separated by $10 \%$ sodium dodecyl sulfate-polyacrylamide gel electrophoresis and transferred to polyvinylidene fluoride membranes. The membranes were first blocked with $5 \%(\mathrm{w} / \mathrm{v})$ nonfat milk in Tris-buffered saline and Tween 20, and then probed with either rabbit polyclonal SOX4 (1:2,000 dilution; catalog no. 17919-1-AP; ProteinTech Group, Inc., Chicago, IL, USA) or rabbit monoclonal GAPDH (1:5,000 dilution; product no. 2118L; Cell Signaling Technology, Inc., Danvers, MA, USA) primary antibodies at $4^{\circ} \mathrm{C}$ overnight. Subsequent to washing 4 times with Tris-buffered saline and Tween 20, the membranes were incubated with the horseradish peroxidase-conjugated secondary antibodies (product no. 7074; Cell Signaling Technology, Inc.) for $1 \mathrm{~h}$ at room temperature. The signals were detected using an enhanced chemiluminescence detection kit (Thermo Fisher Scientific, Inc.).

Plasmid construction and transfection. SOX4-targeting DNA sequences (sense, 5'-GATCCGCGACAAGATCCCTTTCAT TTCAAGAGAATGAAAGGGATCTTGTCGCTGA-3' and antisense, 5'-AGCTTCAGCGACAAGATCCCTTTCATT CTCTTGAAATGAAAGGGATCTTGTCGCG-3') were synthesized, annealed and inserted into pSilencer 4.1-CMV expression vectors (Ambion; Thermo Fisher Scientific, Inc.). KYSE410 and KYSE510 cells were seeded into 6-well plates and transfected with $1 \mu \mathrm{g} /$ well short hairpin RNA (shRNA) or pSilencer 4.1 CMV empty vector plasmids in Lipofectamine 2000 reagent (Invitrogen; Thermo Fisher Scientific, Inc.) to produce shSOK4 and vector cell groups, respectively. Culture medium containing $100 \mu \mathrm{g} / \mathrm{ml}$ geneticin (G418; Beyotime Institute of Biotechnology) was used to grow the cells for 2 weeks at $37^{\circ} \mathrm{C}$ to select for stably transfected cells.

Colony forming assay and SA- $\beta$-Gal staining. Cells were seeded into 6-well plates at 200 cells/well and cultured for 10 days at $37^{\circ} \mathrm{C}$. The cells were then washed with PBS, fixed in $4 \%$ paraformaldehyde and stained with Coomassie brilliant blue.

Doxorubicin-induced cancer cell senescence was analyzed using a $\beta$-galactosidase staining kit (Beyotime Institute of Biotechnology) following the manufacturer's instructions.

Statistical analysis. Data were presented as the mean \pm standard deviation and analyzed using the Wilcoxon paired $t$-test or Spearman's rank correlation coefficient for analysis of the association between SOX4 and senescence marker expression. Statistical calculations were performed with Graphpad Prism 5.0 (GraphPad Software Inc., San Diego, CA, USA). P<0.05 was considered to indicate a statistically significant difference. 
A

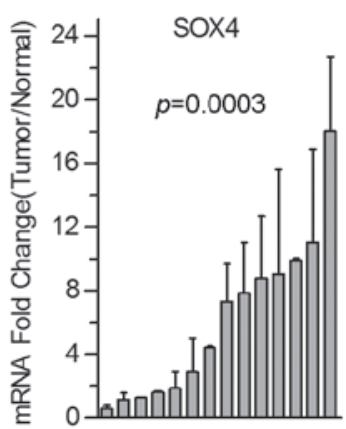

Patients $\mathrm{N}=14$
B

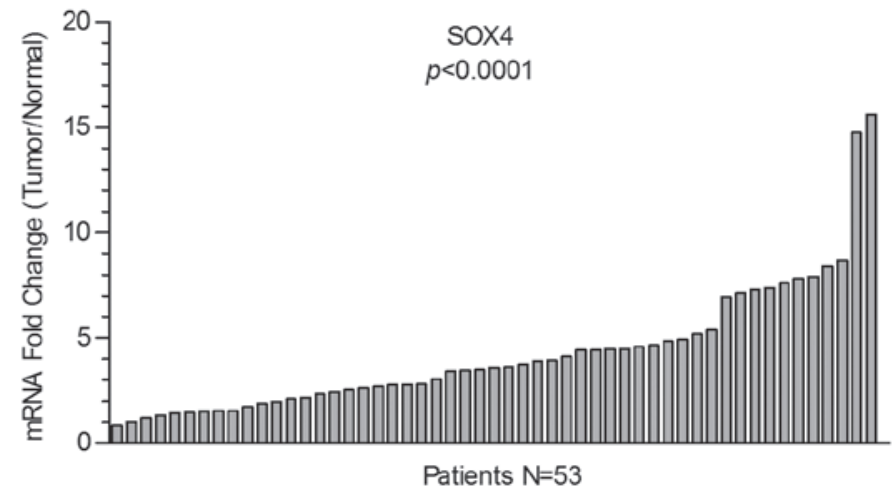

D

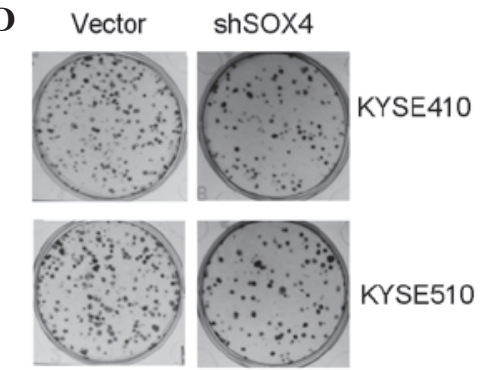

Figure 1. SOX4 expression was upregulated in esophageal squamous cell carcinoma. (A) mRNA levels of SOX4 in 14 pairs of ESCC and non-tumor tissues were determined by reverse transcription-quantitative polymerase chain reaction. (B) mRNA levels of SOX4 in 53 cases of ESCC were obtained from the GSE23400 accession of the NCBI Gene Expression Omnibus database. Fold changes of mRNA were calculated as robust multi-array average $(\mathrm{RMA})_{\text {Tumor }} / \mathrm{RMA}_{\mathrm{Norma}} 1$. (C) SOX4 expression in KYSE410 and KYSE510 cells was inhibited by SOX4-targeting shRNA. (D) SOX4 knockdown decreased colony forming abilities in KYSE410 and KYSE510 cells. mRNA, messenger RNA; SOX4, gender determining region Y-box 4; shRNA/SOX4, short hairpin RNA/SOX4.

\section{Results}

SOX4 is upregulated in ESCC. Tumor and adjacent non-tumor tissues were collected from 14 ESCC patients from Anyang Tumor Hospital between May 2011 and October 2013, and the expression of SOX4 in tumor and paired non-tumor tissues was compared using RT-qPCR. As shown in Fig. 1A, the messenger RNA (mRNA) levels of SOX4 were upregulated in 92\% patients (13/14 cases), with 64\% (9/14 cases) exceeding normal levels by 2 -fold. The cDNA microarray data deposited at NCBI Gene Expression Omnibus database (accession number, GSE23400) (18), which included 53 ESCC samples and 53 matched normal samples that were collected from the Thoracic Surgery Department of Shanxi Cancer Hospital (Taiyuan, China) between 1998 and 2001, were then examined. The SOX4 levels in tumors were found to be upregulated in 52 cases compared with the normal tissues, with $77 \%$ (41/53 cases) exceeding the normal upregulation by 2 -fold (Fig. 1B). Together, these results demonstrated that SOX4 was significantly upregulated in ESCC.

SOX4 knockdown impairs ESCC cell proliferation. To investigate the function of SOX4 in ESCC, a shRNA to target SOX4 was constructed and transfected it into the two ESCC KYSE410 and KYSE510 cell lines. Following selection with $100 \mu \mathrm{g} / \mathrm{ml}$ of G418 for 2 weeks, two cell lines were obtained in which SOX4 were stably knocked down (Fig. 1C). A colony formation assay was performed, which indicated that decreased SOX4 expression was significantly associated with decreased colony numbers in KYSE410 and KYSE510 cells compared with the vector group (Fig. 1D).

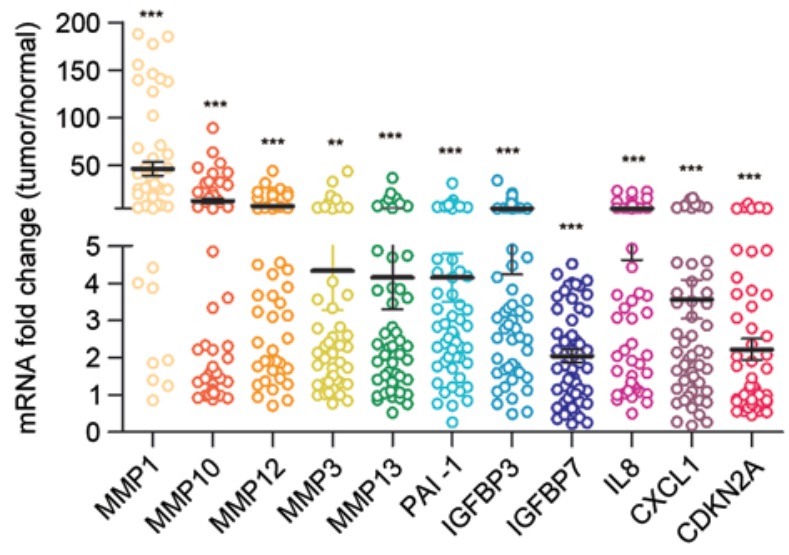

Figure 2. Gene expression profile demonstrated a senescent phenotype in esophageal squamous cell carcinoma. Among the most upregulated genes were numerous molecules used for matrix degradation, chemokine, inflammation factor and other molecules associated with the senescence-associated secretory phenotype (5) ( $\left.\mathrm{P}<0.05 ;{ }^{* *} \mathrm{P}<0.01 ;{ }^{* * *} \mathrm{P}<0.001\right)$. mRNA, messenger RNA; MMP, matrix metalloproteinase; PAI-1, plasminogen activator inhibitor-1; IGF, insulin-like growth factor; IL8, interleukin 8; CXCL1, chemokine (C-X-C motif) ligand 1; CDKN2A, cyclin-dependent kinase inhibitor $2 \mathrm{~A}$.

ESCC has an associated senescent microenvironment. The gene expression profile in ESCC was then examined using the GSE23400 dataset (18). As shown in Fig. 2, among the most upregulated genes in ESCC tumor tissues were numerous matrix-remolding proteinases, including MMP1, MMP10, MMP12, MMP3 and MMP13, chemokines such as CXCL1 and inflammatory factors such as IL8. This gene expression signature had the characteristics of SASP $(5,20,21)$. Other important 

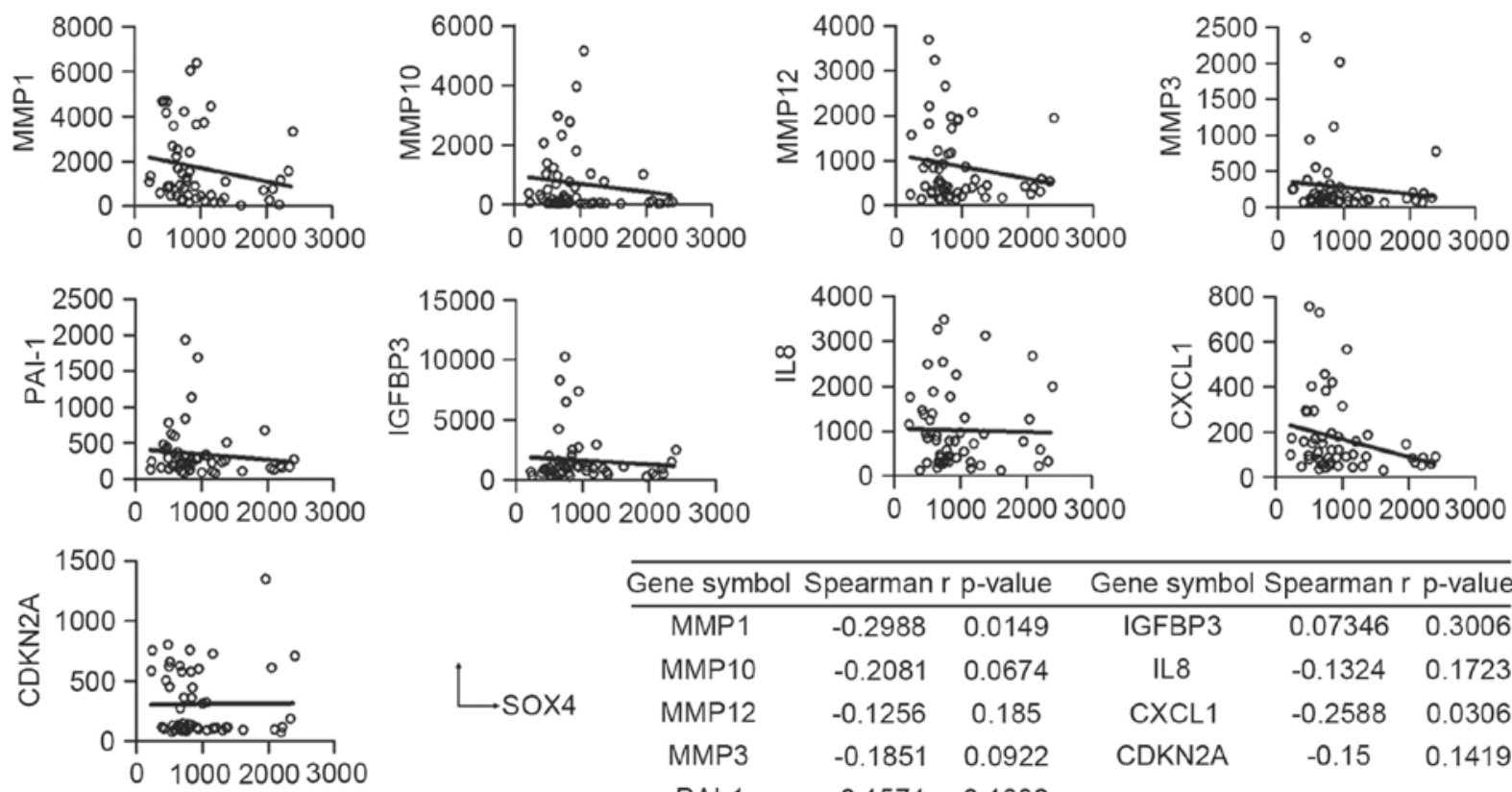

\begin{tabular}{ccccccc} 
& \multicolumn{2}{c}{ Gene symbol Spearman r p-value } & Gene symbol & Spearman r p-value \\
\cline { 2 - 7 } & MMP1 & -0.2988 & 0.0149 & IGFBP3 & 0.07346 & 0.3006 \\
& MMP10 & -0.2081 & 0.0674 & IL8 & -0.1324 & 0.1723 \\
SOX4 & MMP12 & -0.1256 & 0.185 & CXCL1 & -0.2588 & 0.0306 \\
& MMP3 & -0.1851 & 0.0922 & CDKN2A & -0.15 & 0.1419 \\
& PAl-1 & -0.1574 & 0.1302 & & & \\
\hline
\end{tabular}

Figure 3. Gender determining region Y-box 4 expression was reversely correlated with senescence-associated molecules. MMP, matrix metalloproteinase; PAI-1, plasminogen activator inhibitor-1; IGFBP3, insulin-like growth factor-binding protein 3; IL8, interleukin 8; CXCL1, chemokine (C-X-C motif) ligand 1; CDKN2A, cyclin-dependent kinase inhibitor 2A.
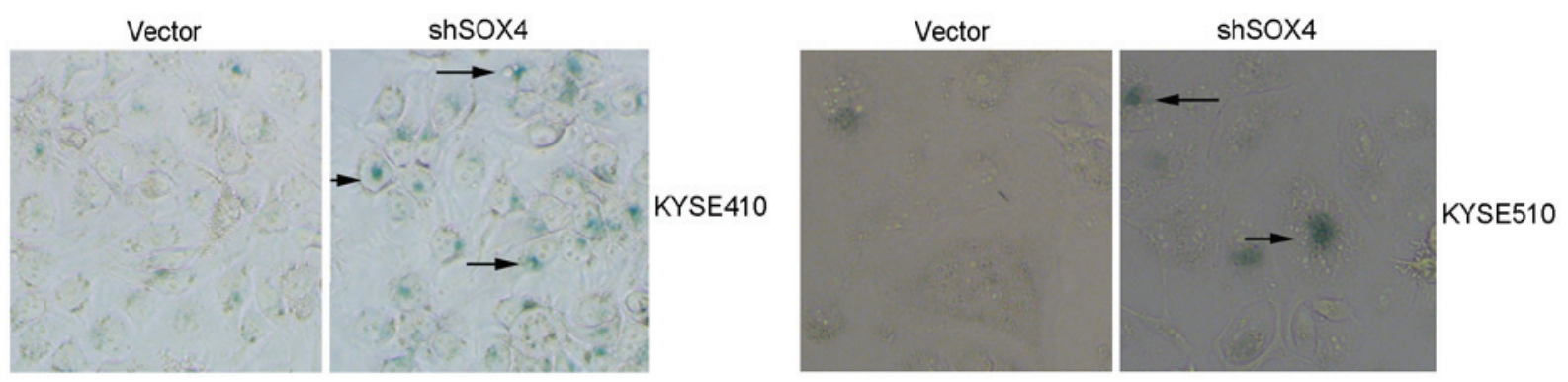

Figure 4. Gender determining region Y-box 4 knockdown enhanced doxorubicin-induced senescence in esophageal squamous cell carcinoma cells. Arrows indicate senescence-associated $\beta$-galactosidase-positive cells.

A

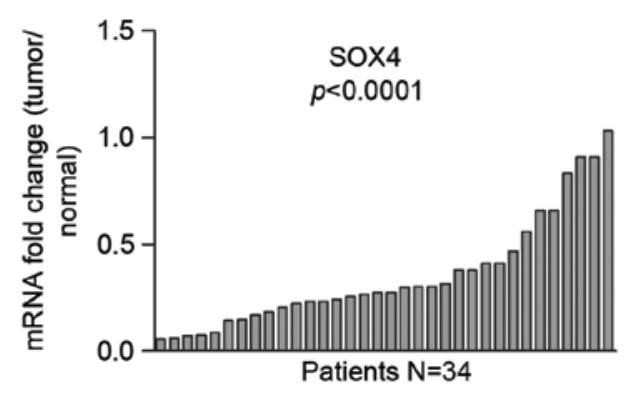

B

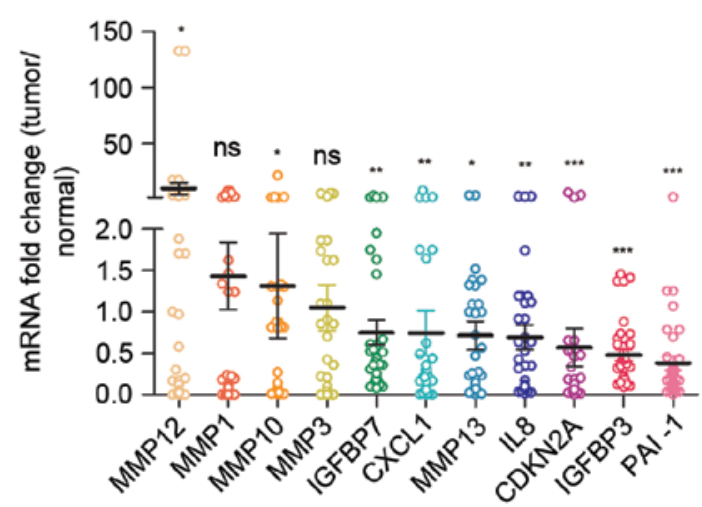

Figure 5. Senescent phenotype was absent in stomach adenocarcinoma. mRNA levels of (A) SOX4 and (B) senescence markers were downregulated in stomach adenocarcinoma. Fold changes were calculated as reads per kilobase per million mapped reads (RPKM) $)_{\text {Tumor }} / \mathrm{RPKM}_{\text {Normal }}\left({ }^{*} \mathrm{P}<0.05 ;{ }^{* * *} \mathrm{P}<0.01 ;{ }^{* * *} \mathrm{P}<0.001\right)$. ns, not significant; mRNA, messenger RNA; SOX4, gender determining region Y-box 4; MMP, matrix metalloproteinase; IGFBP, insulin-like growth factor-binding protein; CXCL1, chemokine (C-X-C motif) ligand 1; IL8, interleukin 8; CDKN2A, cyclin-dependent kinase inhibitor 2A; PAI-1, plasminogen activator inhibitor-1.

senescence mediators such as PAI-1, IGF-binding proteins (IGFBPs) and cyclin-dependent kinase inhibitor 2A (CDKN2A) were also upregulated in ESCC tumor tissues (21). These data suggested the senescent microenvironment in ESCC. 
SOX4 is inversely correlated with senescence markers. To investigate the role of SOX4 in the senescent phenotype of ESCC, Spearman's rank correlation coefficient analysis was performed to assess the association between SOX4 and senescence markers. Fig. 3 shows that in ESCC tumor tissues, the mRNA levels of SOX4 were inversely correlated with the levels of MMP1 ( $\mathrm{P}=0.0149)$, MMP10 ( $\mathrm{P}=0.0674), \mathrm{MMP} 12$, MMP3, PAI-1, IL8, CXCL1 $(\mathrm{P}=0.0306)$ and CDKN2A $(\mathrm{P}=0.1419)$. These results suggested that $\mathrm{SOX} 4$ may have an antisenescence function in ESCC.

SOX4 knockdown promotes doxorubicin-induced senescence in ESCC cells. In vitro-cultured cancer cells do not show senescent phenotypes under normal conditions (20). However, under stresses such as anticancer drug treatment and ionizing radiation, various cancer cells undergo senescence. To further elucidate the antisenescence function of SOX4, KYSE410 and KYSE510 cells that were transfected with SOX4-targeting shRNA or empty vector were treated with $50 \mathrm{ng} / \mathrm{ml}$ doxorubicin for $48 \mathrm{~h}$. The cells were then maintained in drug-free medium for another $72 \mathrm{~h}$ to enter growth-arrest states, and subsequently stained with SA- $\beta$-Gal. In Fig. 4 , the SOX4 knockdown groups showed stronger staining with SA- $\beta$-Gal compared with the control groups in the KYSE410 and KYSE510 cell lines. These results demonstrated the important role of SOX4 in doxorubicin-induced senescence in ESCC cells.

Senescent microenvironment is absent in stomach adenocarcinoma. The TCGA database included the RNA-Seq data of 34 paired tumor and normal tissues of stomach adenocarcinoma and 8 paired tumor and normal tissues of esophageal adenocarcinoma. The present study then investigated whether the senescence-associated gene expression signature exist in stomach adenocarcinoma. The mRNA level of SOX4 was found to be downregulated in stomach adenocarcinomas (Fig. 5A). Although the MMP12 and MMP10 were significantly upregulated in stomach tumor tissues, the mRNA levels of other senescence markers were downregulated (IGFBP7, CXCL1, MMP13, IL8, CDKN2A, IGFBP3 and PAI-1) or not significantly changed (MMP1 and MMP3) in tumor tissues compared with normal tissues (Fig. 5B). Hence, the senescent microenvironment was not evidently present in stomach adenocarcinoma.

\section{Discussion}

Senescent cells are growth-arrested, but remain metabolically active and can develop a secretory profile named SASP (4). Senescence is considered to limit the expansion of early neoplastic cells (5) and is, therefore, a potent cancer-protective response to oncogenic events. However, the direct evidence of senescence in cancer tissues is limited (22). Through open-data mining, a plethora of senescence-associated molecules, which included proteinases, chemokines and inflammatory factors, were found to be upregulated in ESCC tumor tissues in the present study. Although the origin of these senescence makers could not be determined by microarray assays, this gene expression signature showed the characteristic of senescence in the ESCC microenvironment. The senescence-associated gene expression profile was not shown in stomach adenocarcinoma, suggesting that the senescent phenotype may be organ specific. Previous studies identified various oncogenes that mutated in ESCC, including tumor protein 53 (p53), RB transcriptional corepressor 1, CDKN2A, phosphatidylinositol-4,5-bisphosphate 3-kinase catalytic subunit $\alpha$ and NOTCH1 (23). Deregulated oncogenes are well-known inducers of cellular senescence (9), and may contribute to the senescent phenotype in ESCC.

The present study demonstrated that SOX4 is upregulated in ESCC and that SOX4 expression is inversely correlated with a cohort of senescence markers. SOX4 knockdown decreased cell proliferation and enhanced doxorubicin-induced cellular senescence in vitro. These results suggested the important role of SOX4 in senescence evading in ESCC. Indeed, Foronda et al recently reported that mice with reduced whole-body SOX4 expression displayed accelerated aging and reduced cancer incidence, highlighting the crucial roles of SOX4 in cancer progression (24). Pan et al (25) revealed that SOX4 was upregulated in response to DNA damage, and stabilized p53 protein by blocking mouse double minute 2 homolog-mediated p53 ubiquitination and degradation. Whether the p53 pathway is required for the antisenescence function of SOX4 requires additional investigation.

In conclusion, the current results revealed the protective role of SOX4 in cancer cell senescence. As senescence evading is mechanistically implicated in cancer development and refractory disease, we propose SOX4 targeting as a potential method of cancer prevention and treatment in the future. However, whether SOX4 has a similar role in cell senescence accompanied by chronological aging is a topic that requires further investigation.

\section{Acknowledgements}

The present study was, in part, supported by the US-Chinese Anti-Cancer Association (Natural Science Foundation of China, Beijing, China; grant nos. 81071655,81372149,91229115 and 81272251), the China Postdoctoral Science Foundation (Beijing, China; grant no. 2015M572366), the Innovation of Science and Technology Commission of Shenzhen Municipality (Shenzhen, China; grant no. JCYJ20130329102515481) and the Key Laboratory Project of Shenzhen (Shenzhen, China; grant no. ZDSY20130329101130496).

\section{References}

1. Kamangar F, Dores GM and Anderson WF: Patterns of cancer incidence, mortality, and prevalence across five continents: Defining priorities to reduce cancer disparities in different geographic regions of the world. J Clin Oncol 24: 2137-2150, 2006.

2. Zhang SK, Guo LW, Chen Q, Zhang M, Liu SZ, Quan PL, $\mathrm{Lu}$ JB and Sun XB: Prevalence of human papillomavirus 16 in esophageal cancer among the Chinese population: A systematic review and meta-analysis. Asian Pac J Cancer Prev 15: 10143-10149, 2014.

3. Xu Y, Yu X, Chen Q and Mao W: Neoadjuvant versus adjuvant treatment: Which one is better for resectable esophageal squamous cell carcinoma? World J Surg Oncol 10: 173, 2012.

4. Pérez-Mancera PA, Young AR and Narita M: Inside and out: The activities of senescence in cancer. Nat Rev Cancer 14: 547-558, 2014.

5. Kuilman T and Peeper DS: Senescence-messaging secretome: SMS-ing cellular stress. Nat Rev Cancer 9: 81-94, 2009. 
6. Eren M, Boe AE, Murphy SB, Place AT, Nagpal V, Morales-Nebreda L, Urich D, Quaggin SE, Budinger GR, Mutlu GM, et al: PAI-1-regulated extracellular proteolysis governs senescence and survival in Klotho mice. Proc Natl Acad Sci USA 111: 7090-7095, 2014.

7. Coppé JP, Patil CK, Rodier F, Krtolica A, Beauséjour CM, Parrinello S, Hodgson JG, Chin K, Desprez PY and Campisi J: A human-like senescence-associated secretory phenotype is conserved in mouse cells dependent on physiological oxygen. PLoS One 5: e9188, 2010.

8. Kuilman T, Michaloglou C, Vredeveld LC, Douma S, van Doorn R, Desmet CJ, Aarden LA, Mooi WJ and Peeper DS: Oncogene-induced senescence relayed by an interleukin-dependent inflammatory network. Cell 133: 1019-1031, 2008.

9. MooiWJand PeeperDS: Oncogene-inducedcell senescence-halting on the road to cancer. N Engl J Med 355: 1037-1046, 2006.

10. Vervoort SJ, van Boxtel R and Coffer PJ: The role of SRY-related HMG box transcription factor 4 (SOX4) in tumorigenesis and metastasis: Friend or foe? Oncogene 32: 3397-3409, 2013.

11. Penzo-Méndez AI: Critical roles for SoxC transcription factors in development and cancer. Int J Biochem Cell Biol 42: 425-428, 2010.

12. Zhang J, Liang Q, Lei Y, Yao M, Li L, Gao X, Feng J, Zhang Y, Gao H, Liu DX, et al: SOX4 induces epithelial-mesenchymal transition and contributes to breast cancer progression. Cancer Res 72: 4597-4608, 2012.

13. Hur W, Rhim H, Jung CK, Kim JD, Bae SH, Jang JW, Yang JM, Oh ST, Kim DG, Wang HJ, et al: SOX4 overexpression regulates the p53-mediated apoptosis in hepatocellular carcinoma: Clinical implication and functional analysis in vitro. Carcinogenesis 31: 1298-1307, 2010.

14. Lin CM, Fang CL, Hseu YC, Chen CL, Wang JW, Hsu SL, Tu MD, Hung ST, Tai C, Uen YH and Lin KY: Clinical and prognostic implications of transcription factor SOX4 in patients with colon cancer. PLoS One 8: e67128, 2013.

15. Ramezani-Rad P, Geng H, Hurtz C, Chan LN, Chen Z, Jumaa H, Melnick A, Paietta E, Carroll WL, Willman CL, et al: SOX4 enables oncogenic survival signals in acute lymphoblastic leukemia. Blood 121: 148-155, 2013.
16. Scharer CD, McCabe CD, Ali-Seyed M, Berger MF, Bulyk ML and Moreno CS: Genome-wide promoter analysis of the SOX4 transcriptional network in prostate cancer cells. Cancer Res 69: 709-717, 2009

17. Tiwari N, Tiwari VK, Waldmeier L, Balwierz PJ, Arnold P, Pachkov M, Meyer-Schaller N, Schübeler D, van Nimwegen E and Christofori G: Sox4 is a master regulator of epithelial-mesenchymal transition by controlling Ezh2 expression and epigenetic reprogramming. Cancer Cell 23: 768-783, 2013.

18. $\mathrm{Su} \mathrm{H}, \mathrm{Hu} \mathrm{N}$, Yang $\mathrm{HH}$, Wang $\mathrm{C}$, Takikita M, Wang $\mathrm{QH}$, Giffen C, Clifford R, Hewitt SM, Shou JZ, et al: Global gene expression profiling and validation in esophageal squamous cell carcinoma and its association with clinical phenotypes. Clin Cancer Res 17: 2955-2966, 2011.

19. Livak KJ and Schmittgen TD: Analysis of relative gene expression data using real-time quantitative PCR and the 2(-Delta Delta C(T)) Method. Methods 25: 402-408, 2001.

20. Elzi DJ, Lai Y, Song M, Hakala K, Weintraub ST and Shiio Y: Plasminogen activator inhibitor 1-insulin-like growth factor binding protein 3 cascade regulates stress-induced senescence. Proc Natl Acad Sci USA 109: 12052-12057, 2012.

21. Coppé JP, Desprez PY, Krtolica A and Campisi J: The senescence-associated secretory phenotype: The dark side of tumor suppression. Annu Rev Pathol 5: 99-118, 2010.

22. Collado M and Serrano M: Senescence in tumours: Evidence from mice and humans. Nat Rev Cancer 10: 51-57, 2010.

23. Song Y, Li L, Ou Y, Gao Z, Li E, Li X, Zhang W, Wang J, $\mathrm{Xu}$ L, Zhou Y, et al: Identification of genomic alterations in oesophageal squamous cell cancer. Nature 509: 91-95, 2014

24. Foronda M, Martínez P, Schoeftner S, Gómez-López G, Schneider R, Flores JM, Pisano DG and Blasco MA: Sox4 links tumor suppression to accelerated aging in mice by modulating stem cell activation. Cell Rep 8: 487-500, 2014

25. Pan X, Zhao J, Zhang WN, Li HY, Mu R, Zhou T, Zhang HY, Gong WL, Yu M, Man JH, et al: Induction of SOX4 by DNA damage is critical for p53 stabilization and function. Proc Natl Acad Sci USA 106: 3788-3793, 2009. 